\title{
Mediating Effect of Deteriorated Kidney Function in Comorbidity-related Excess Deaths in COVID-19: A Retrospective Cohort Study
}

\section{Dongling Luo}

Sun Yat-Sen University

Qiaomei Liu

Wuhan University

Changming Xie

Sun Yat-Sen University

\section{Weidong Wang}

Sun Yat-sen University Zhongshan School of Medicine

\section{Xiong Wang}

Wuhan University

Juan Wu

Wuhan University

Maomao Xi

Wuhan University

\section{Yilin Yin}

Wuhan University

Di Xiong

Wuhan University

\section{Yuwei Wu}

Wuhan University

\section{Yuqing Xie}

Wuhan University

\section{Ximin Shi}

Wuhan University

\section{Pengcheng Luo}

Wuhan University

\section{Fang Dong}

Wuhan University

Hui Huang ( $\nabla$ huangh8@mail.sysu.edu.cn )

The Eighth Affliated Hospital, Sun Yat-sen University https://orcid.org/0000-0001-5716-4012 
Keywords: COVID-19, kidney function, comorbidities, in-hospital death, mediation effect

Posted Date: October 7th, 2020

DOI: https://doi.org/10.21203/rs.3.rs-85393/v1

License: (a) (i) This work is licensed under a Creative Commons Attribution 4.0 International License. Read Full License 


\section{Abstract}

\section{Background}

In-hospital death risks vary in COVID-19 patients with comorbidities. Kidney function decline is prevalent in this course and found associated with in-hospital death. However, what role it plays is not clear.

\section{Methods}

To explore the exact role of deteriorated kidney function, we applied a retrospective cohort study including 1266 participants in Wuhan Tongren Hospital between January 27 and March 3, 2020. Demographic characteristics, preexisting comorbidities history, organ function data and outcomes were extracted. Deteriorated kidney function was identified as the decline percentage, assessed by an increase in peak serum creatinine from the baseline. Mediating effect was calculated by mediation analysis.

Key Results

1266 hospitalized COVID-19 patients ( $60 \pm 15$ years, $47.8 \%$ are male) were included, with an overall in-hospital death rate of $4.42 \%(56 / 1266)$. For critical cases, $77.02 \%$ had at least one preexisting comorbidity. Patients with comorbidities suffered higher in-hospital death and more severe decline of kidney function. Compared to patients with minor function decline $(<10 \%)$, significant risk increase was found in those with more severe one (OR $3.57 ; 95 \% \mathrm{Cl} 1.70$ to $7.52 ; \mathrm{P}=.001$ for moderate with $10-50 \%$ decline, and $37.45 ; 95 \% \mathrm{Cl} 18.71$ to $74.55 ; \mathrm{P}$ $\mathbb{\nabla} .001$ for severe with $>50 \%$ ). More interestingly, the mediation analysis found deteriorated kidney function played as an important mediator between different comorbidities and COVID-19 patients' in-hospital death, with the mediation effect of $11 \%, 12 \%, 16 \%$ and $32 \%$ respectively for hypertension, chronic obstructive pulmonary disease, cardiovascular disease and chronic kidney disease.

\section{Conclusions}

All-cause deteriorated kidney function is strongly associated with increase of in-hospital death in COVID-19 and partially mediates the facilitating effect of preexisting comorbidities on in-hospital death. Thus, dynamic monitoring kidney function, preventing the deterioration of kidney function might be helpful to improve survival in COVID-19 patients, especially those with preexisting comorbidities.

\section{Introduction}

Preexisting comorbidities are prevalent among COVID-19 patients, especially in critical cases. According to a most last case series in Canada, at least one comorbidity was present in $73.5 \%$ COVID-19 patients who was admitted to intensive care unit (ICU) (1). The in-hospital death rates of COVID-19 patients are reported to range from $0.4 \%$ to $49.0 \%$, which is correlated with preexisting comorbidities (2-4). As reported in a recent metaanalysis, hypertension, diabetes, cardiovascular disease (CVD) were independent risk factors for poor prognosis in COVID-19 patients, while no correlation was found in renal or liver disease (5). However, in another study reported in Iran, the death rate was higher in chronic kidney disease (CKD) patients $(16.66 \%)$ than those with CVD (10.81\%), hypertension (13.55\%) or diabetes (9.73\%) (6). Few studies have looked into this inconformity and the underlying mechanisms on how these preexisting comorbidities accelerate this 
process. Immune dysregulation and prolonged inflammation were regarded as possible mechanisms (2). However, none of them gave a detailed inspection.

Emerging data have reported that patients with preexisting comorbidities were more likely to suffer acute organ injury (7-9), which has been recently implicated as an independent risk for increased in-hospital death in COVID-19 patients (10-12). As reported by T. Guo, 27.8\% of the hospitalized COVID-19 patients exhibited myocardial injury (8) while up to $75 \%$ of the COVID-19 patients experienced different degree of kidney function decline (13). However, the exact role of this universal alternation has not been figured out.

Based on these, we hypothesized that comorbidities facilitated excess deaths by causing renal damage. To this end, we here studied retrospectively 1266 COVID-19 patients in Wuhan. Logistic analysis was applied to prove the facilitating role of deteriorated kidney function for in-hospital death in COVID-19 patients with various comorbidities. What's more, it was determined as an important mediator in comorbidities related death, which gave a clue of COVID-19 patients' management.

\section{Methods}

\section{Study design and participants}

We retrospectively collected data from 1276 patients with laboratory-confirmed COVID-19 infection who were treated at Tongren Hospital of Wuhan University between January 27 and March 3, 2020. A confirmed case of COVID-19 was defined by a positive result on a reverse-transcriptase-polymerase-chain-reaction (RT-PCR) assay of nasal or pharyngeal swab specimens $(14,15)$. De-identified data on patients' demographic characteristics, preexisting comorbidities history, laboratory tests and outcome status were extracted from the electronic medical records. Patients with missing value for baseline serum creatinine or lack of repeated measurements of creatinine were excluded in this study (10, in total). All data were checked by two physicians (LQM and WX) individually. The study protocol conformed to the ethical guidelines of the 1975 Declaration of Helsinki and was approval by the Ethics Committee of Tongren Hospital of Wuhan University (KY2020-020). Owing to the rapid emergence of this infectious disease, written informed consent was waived.

\section{Data collection}

Clinical information included age, sex, history of smoking and preexisting comorbidities as noted in either the inpatient or the outpatient electronic health record. Comorbidities, including hypertension, underlying CVD, CKD, diabetes mellitus, chronic liver disease or chronic obstructive pulmonary disease (COPD) were recorded. The presence of comorbidities was based on patients' self-report on admission or identified from the documented electronic health record. Routine blood examinations, serum biochemical tests (including indicators for different organ function), and infection-related indicators such as C-reactive protein were collected. Repeated serum creatinine during hospitalization were collected for further evaluation.

\section{Study definitions}

The severity of COVID-19 was classified into critical and non-critical cases, according to the management guideline for COVID-19(16, 17). Patients were considered as critical cases if they met one of the following 
criteria: 1) respiratory failure requiring mechanical ventilation; 2) presence of shock; 3 ) other organ failure requiring ICU admission $(16,17)$. The requirement for ICU admission during hospitalization was judged by the treating physicians. The main endpoint in our study was in-hospital death, defined as patients' documented death for any cause during hospitalization.

Deterioration of kidney function was identified as a relative increase in serum creatinine (18), assessed by an increase in peak serum creatinine from baseline during hospitalization in our study. Baseline serum creatinine was defined as the serum creatinine value on admission while the peak was the highest creatinine level during hospitalization. The severity of decline of kidney function was classified into minor, moderate and severe (respectively $<10 \%, 10-50 \%$ and $>50 \%$ decline in kidney function).

\section{Statistical analysis}

Continuous variables were presented as means and standard deviations (SD) or medians and interquartile ranges (IQR) as appropriate, and the categorical variables were presented as counts and percentages. Twosample t test or Wilcoxon rank-sum test were used for continuous variables and chi-square test for categorical variables. Univariate logistic regression analysis was performed to ascertain the effects of comorbidities and deterioration of kidney function on the likelihood of negative outcomes. Odds ratios (ORs) and corresponding $95 \%$ confidence interval $(\mathrm{Cl})$ were calculated. These models were then adjusted for age and gender (model 2) and further adjusted for white blood cell counts, smoking status and baseline serum creatinine in model 3 , as these were generally accepted risk factors associated with increased mortality $(2,19)$.

Further, simple mediation models were sought to explain the mechanism underlying the observed relationship between those independent variables (individual comorbidities, i.e. hypertension, CVD, CKD, diabetes, liver disease or COPD) and dependent variable (in-hospital death) via the hypothetical mediator (deteriorated kidney function). It also allowed us to quantify the proportion of mediating effect with respect to the total effect of individual comorbidities on mortality risk. Different paths were created in this model: pathway a, the effect of individual comorbidities on deterioration of kidney function; pathway $b$, the effect of deterioration of kidney function on in-hospital death in COVID-19 patients by the mediator included; pathway c, total effect of individual comorbidities on in-hospital death in COVID-19 patients; and pathway c', the remaining effect of comorbidities on in-hospital death not mediated by deteriorated kidney function. Bootstrapping methods were used for testing the mediation hypotheses using a resample procedure of 1000 bootstrap samples, as recommended by Preacher and Hayes (20). Point estimates and $95 \% \mathrm{Cl}$ were calculated. The point estimate was considered to be significant when the confidence interval did not contain zero.

All statistical analyses were performed using Stata/SE 15.1 software (Stata Corp, College Station, TX, USA), unless otherwise indicated. All $P$ values $<0.05$ were considered statistically significant.

\section{Results}

\section{Demographic and clinical characteristics of COVID-19 patients}

1276 hospitalized patients were enrolled initially. Ten of them were excluded for missing value. The mean (SD) age was 60.31 (14.99) years, and 47.87\% (606/1266) were male. Totally, 148 out of 1266 patients $(11.69 \%)$ 
were diagnosed as critical cases while the ICU admission rate was $6.79 \%$ (86/1266). The overall in-hospital death rate was $4.42 \%(56 / 1266)$. Detailed information of other characteristics is presented in Table 1.

\section{Comorbidities were prevalent in COVID-19 patients and associated with higher in-hospital death.}

$56.16 \%(711 / 1266)$ had at least one type of preexisting comorbidities, to be more detailed, hypertension (34.12\%), CVD (16.43\%), CKD (6.56\%), diabetes (14.77\%), liver disease $(8.45 \%)$ or COPD (15.17\%). For critical cases $(67 \pm 14$ years), $77.02 \%$ have at least one preexisting comorbidity. As presented in Figure 1, patients with these comorbidities suffered excess death (6.71\% for hypertension, $15.87 \%$ for CVD, $19.28 \%$ for CKD, $8.02 \%$ for diabetes, $9.35 \%$ for liver disease and $23.96 \%$ for COPD). The same conclusion can be drawn, when other indicators of illness severity was tested, including critical cases and ICU admission (Table 2). It's revealed that preexisting CVD, CKD, COPD or liver diseases were at higher risk of in-hospital death. The odds ratios were highest for COPD (OR 44.70, 95\%, Cl 20.71-96.48), followed by CKD (OR 7.08, 95\% Cl 3.55-14.13) and CVD (OR $6.12,95 \% \mathrm{Cl} 3.35-11.19)$. Of note, we did observe excess death in patients with hypertension and diabetes, but this did not achieve statistical significance (Table 2).

\section{Deteriorated kidney function was remarkable in patients with comorbidities and progressively correlated with increased risk of in-hospital death}

Generally, COVID-19 patients suffered kidney impairment during hospitalization. The average decline in kidney function was much notable in non-survivors ( $1.13 \pm 1.84$ vs $0.06 \pm 0.32$ ). More specifically, the majority of the survivors had less than $10 \%$ decline of kidney function compared to the non-survivors ( $85.62 \%$ vs $39.29 \%$ ) while nearly half of the non-survivors suffered more than $50 \%$ decline of kidney function $(41.07 \%$ vs $2.4 \%)$. As presented in Figure 2A, COVID-19 patients with hypertension, CVD, CKD, diabetes, liver diseases or COPD had correspondingly higher prevalence of more severe kidney function decline. In addition, with similar degree of kidney function decline, the in-hospital death rates were many-fold higher in those with comorbidities (Figure 2B). It suggested that the impact of deteriorated kidney function was more detrimental to those with comorbidities.

Next, we applied a univariate model to examine the association between deteriorated kidney function and excess death. As expected, a graded, progressive association was observed. Generally, there was a 4.3-fold increase in the odds of in-hospital death per unit decline in kidney function. For moderate kidney decline patients, the odds was 3.57 -fold higher when compared to the minor ones. And the increase risk was more striking for severe ones (OR $37.45,95 \% \mathrm{Cl} 18.71-74.55)$. These results remained significant even after adjustment for age, gender (model 2), and further for smoking status, white blood cell count and baseline serum creatinine (model 3, seen in Figure 3). Interestingly, compared to preexisting CKD, deteriorated kidney function applied much higher risk of death in other types of comorbidities (seen in Appendix eFigure 1).

\section{The association of comorbidities and excess deathin COVID-19 patients was partially mediated by deteriorated kidney function.}

The components analyzed in the mediation model are shown in Figure 4 and eTable 1(Appendix). Pathway c is the total effect of individual comorbidity on excessdeath. Pathway a represents the association between individual comorbidity and deteriorated kidney function, which revealed that CVD, CKD, diabetes, liver disease 
or COPD were associated with more severe decline in kidney function (Appendix eTable 1). Pathway b represents the association between deteriorated kidney function and excessdeath. After testing the mediation hypothesis using the bootstrapping method, the total proportion of overall effect mediated by deterioration of kidney function was estimated to $11 \%, 16 \%, 19 \%, 21 \%$ and $12 \%$, respectively for hypertension, CVD, diabetes, liver diseases and COPD. While, the mediation effect of deteriorated kidney function for diabetes and liver diseases did not reach a statistical significance. It should be noted that the mediation effect of deterioration of kidney function (32\%) was highest, which indicated it was most crucial to those with CKD.

\section{Discussion}

Our analysis revealed that comorbidities were prevalent in COVID-19. Compared to those without, patients with preexisting CVD, CKD, COPD or liver disease sustained excess death. More importantly, our report highlights for the first time that deteriorated kidney function during hospitalization is a detrimental factor to excess death and partially mediates the facilitating effect of comorbidities, which provides novel insights into comorbidityrelated excess death.

Our study described the characteristics of COVID-19 in a larger population with a wider range of comorbidities. In this study, $56.16 \%$ patients have at least one preexisting comorbidity. This is consistent with another published research in Wuhan (48\%) (21). Although, a lower comorbidity rate (25.1\%) was observed in a larger descriptive research in whole China (2). However, it's surprised nearly $90 \%$ or even above $90 \%$ of the hospitalized COVID-19 patients were with at least one type of comorbidities in the US $(22,23)$. This difference may occur from various admission standards, but generally, comorbidities were quite common for hospitalized COVID-19 patients. For critical cases, comorbidities are even more prevalent; $77.02 \%$ in our research coincides with $73.5 \%$ in Canada (1).

Compared to COVID-19 patients without preexisting comorbidities, those with CVD, CKD, COPD or liver disease had greater excess death. The strength of correlation between different comorbidities and the prognosis, however, was inconsistent when compared with the previous researches $(2,5,24)$. We do not find significant risk increase in hypertension and diabetes. The reasons for these inconsistences are still confusing. Thus, how these comorbidities accelerate in-hospital death is an urge problem remained to be solved.

In the current study, it's shown that patients with comorbidities were more prone to experience deteriorated kidney function. Although there were some publications on kidney involvement and COVID-19 infection, they mainly focused on the phenomenon of higher mortality risk associated with comorbidities or kidney diseases or simply presented the prevalence of kidney injury during the disease course $(5,11,13)$. Whereas, our mediation analysis demonstrates that the deteriorated kidney function exhibited different proportion of mediation effect on the accelerating role of comorbidities. The association of comorbidities and high inhospital death could be partially explained by deteriorated kidney function, with a mediation effect ranging from $11 \%$ to $32 \%$ and highest for CKD. In line with previous findings $(11,13)$, deteriorated kidney function is prevalent in COVID-19 patients and strongly related to higher in-hospital death rate, especially in those with comorbidities. However, previous studies were focusing on acute kidney injury (11ه13). While, in our research, any declines in kidney function were found associated with excess death independent of age, gender, smoking status, white blood cell counts and baseline serum creatinine. Although the highest risk occurred in patients 
with more than $50 \%$ decline of kidney function, those with more than $10 \%$ decline of kidney function ought not to be neglected. However, according to our findings, deteriorated kidney function is not the only mediator in comorbidities related excess death. Meanwhile, more detailed mechanism as well as the cause-effect relationship could not be confirmed for the retrospective nature of this study.

The pathogenesis of COVID-19 related kidney function deterioration and how it accelerates in-hospital death is largely unknown. It's supposed to be related to direct viral infection or dysregulated immune response $(10,25$, 26). SARS-CoV-2 enters cells by binding angiotensin-converting enzyme-2 (ACE2) $(27,28)$. Theoretically, the decrease of ACE2, caused by SARS-CoV-2 infection, would break the balance between ACE and ACE2. As a result, Renin-Angiotensin-Aldosterone system (RAAS) was positively regulated and angiotensin II type 1 receptor was over stimulated, which may further cause inflammation activation, endothelial injury and mitochondrial dysfunction $(29,30)$. The kidney and cardiovascular system are supposed as main victims for they are main targets of angiotensin II. These was astonishingly consistent with our findings, for we get the most mediating effect in CKD and CVD patients. Besides, drug-related kidney injury could be another important factor. According to "COVID-19 Diagnosis and Treatment Protocol of China", many potential nephrotoxic drugs were proposed, including some kind of anti-virus drugs (examples: a-interferon, lopinavir and ribavirin), resochin and Chinese traditional medicine( examples: Angong Niuhuang pill, Tanreqing injection and Xingnaojing injection (16). It may be reasonable to speculate that the deteriorated kidney function could be a sign of renal involvement of SARS-COV-2 infection. And it may be somehow relevant to RAAS, which requires further investigations.

For now, kidney function hasn't been emphasized in COVID-19 patients' management both in China and Occident $(16,17,31)$. These findings provide novel insights into comorbidities-related in-hospital death, which could be helpful in stratifying high-risk patients during this pandemic and advising prevention practices for high-risk patients. In other words, the decline of kidney function is likely considered as an important factor in risk stratification models for COVID-19. Thus, dynamic monitoring the kidney function is of paramount importance in COVID-19 patients, especially in those with preexisting comorbidities. Additionally, avoiding the occurrence of hypotension/hypoxemia or using nephrotoxic drugs, attenuating the synthesis of proinflammatory cytokines might help to lower the risk of jeopardizing the kidney function and improve chances of survival in patients with preexisting comorbidities.

But patients were not followed after discharge in this investigation. Hence, recovery of the kidney function and the full clinical course of these patients could not be further evaluated.

\section{Conclusion}

The alternation of kidney function should be noticed in COVID-19 patients' management for it may work as an important mediator for comorbidities related in-hospital death.

\section{Abbreviations}




\begin{tabular}{ll} 
COVID-19 & Coronavirus Disease 2019 \\
\hline COPD & Chronic Obstructive Pulmonary Disease \\
\hline CVD & Cardiovascular Disease \\
\hline CKD & Chronic Kidney Diseases \\
\hline ICU & Intensive Care Unit \\
\hline CI & Confidence Interval \\
\hline OR & Odds Ratio \\
\hline ACE2 & Angiotensin-Converting Enzyme-2 \\
\hline RAAS & Renin-Angiotensin-Aldosterone system
\end{tabular}

\section{Declarations}

\section{Ethics Approval and Consent to Participate}

The study protocol conformed to the ethical guidelines of the 1975 Declaration of Helsinki and was approval by the Ethics Committee of Tongren Hospital of Wuhan University (KY2020-020). Owing to the rapid emergence of this infectious disease, written informed consent was waived.

\section{Consent for publication}

Not applicable

\section{Availability of supporting data}

The datasets used and/or analysed during the current study are available from the corresponding author on reasonable request

\section{Competing interests}

The authors declare that they have no competing interests.

\section{Funding}

This work was supported by National Natural Science Foundation of China [81870506, 81670676 and 81422011], project of traditional Chinese medicine in Guangdong province [20201062], Basic Research Project of Shenzhen Science and Technology Innovation Committee [JCYJ20180306174648342 and JCYJ20190808102005602], Shenzhen Futian District Public Health Research Project[FTWS2019003] to Hui Huang and National Natural Science Foundation of China (81770688), Leading Medical Talents in Hubei Province to Pengcheng Luo.

\section{Authors' contributions}


LDL, LPC, DF and HH designed the study; LQM, WX, WJ, XMM, YYL, XD, WYW, XYQ and SXM performed the data extraction; XCM, LDL, LQM, WX, WJ, XMM, YYL, XD, WYW, XYQ and SXM analyzed and interpreted the data; LDL, XCM, WWD, LPC, DF and HH drafted and revised the paper; all authors approved the final version of the manuscript.

\section{Acknowledgements}

Not applicable

\section{Authors' information}

Not applicable

\section{References}

1. Mitra AR, Fergusson NA, Lloyd-Smith E, et al. Baseline characteristics and outcomes of patients with COVID-19 admitted to intensive care units in Vancouver, Canada: a case series [published online ahead of print, 2020 May 27]. CMAJ. 2020;cmaj.200794. doi:10.1503/cmaj.200794

2. Guan WJ, Liang WH, Zhao Y, et al. Comorbidity and its impact on 1590 patients with COVID-19 in China: a nationwide analysis. Eur Respir J. 2020;55(5):2000547. Published 2020 May 14. doi:10.1183/13993003.00547-2020

3. Porcheddu R, Serra C, Kelvin D, Kelvin N, Rubino S. Similarity in Case Fatality Rates (CFR) of COVID19/SARS-COV-2 in Italy and China. J Infect Dev Ctries. 2020;14(2):125-128. Published 2020 Feb 29. doi:10.3855/jidc. 12600

4. Wu Z, McGoogan JM. Characteristics of and Important Lessons From the Coronavirus Disease 2019 (COVID-19) Outbreak in China: Summary of a Report of 72314 Cases From the Chinese Center for Disease Control and Prevention [published online ahead of print, 2020 Feb 24]. JAMA. 2020;10.1001/jama.2020.2648. doi:10.1001/jama.2020.2648

5. Wang B, Li R, Lu Z, Huang Y. Does comorbidity increase the risk of patients with COVID-19: evidence from meta-analysis. Aging (Albany NY). 2020;12(7):6049-6057. doi:10.18632/aging.103000

6. Nikpouraghdam M, Jalali Farahani A, Alishiri G, et al. Epidemiological characteristics of coronavirus disease 2019 (COVID-19) patients in IRAN: A single center study. J Clin Virol. 2020; 127:104378. doi:10.1016/j.jcv.2020.104378

7. Yang $\mathrm{X}, \mathrm{Yu} \mathrm{Y}, \mathrm{Xu} \mathrm{J}$, et al. Clinical course and outcomes of critically ill patients with SARS-CoV-2 pneumonia in Wuhan, China: a single-centered, retrospective, observational study [published correction appears in Lancet Respir Med. 2020 Apr;8(4):e26]. Lancet Respir Med. 2020;8(5):475-481. doi:10.1016/S2213-2600(20)30079-5

8. Guo T, Fan Y, Chen M, et al. Cardiovascular Implications of Fatal Outcomes of Patients With Coronavirus Disease 2019 (COVID-19) [published online ahead of print, 2020 Mar 27]. JAMA Cardiol. 2020; e201017. doi:10.1001/jamacardio.2020.1017

9. Dariya B, Nagaraju GP. Understanding novel COVID-19: Its impact on organ failure and risk assessment for diabetic and cancer patients [published online ahead of print, 2020 May 6]. Cytokine Growth Factor Rev. 
2020; S1359-6101(20)30078-2. doi:10.1016/j.cytogfr.2020.05.001

10. Durvasula R, Wellington T, McNamara E, Watnick S. COVID-19 and Kidney Failure in the Acute Care Setting: Our Experience From Seattle [published online ahead of print, 2020 Apr 8]. Am J Kidney Dis. 2020; S0272-6386(20)30618-1. doi:10.1053/j.ajkd.2020.04.001

11. Cheng Y, Luo R, Wang K, et al. Kidney disease is associated with in-hospital death of patients with COVID19. Kidney Int. 2020;97(5):829-838. doi:10.1016/j.kint.2020.03.005

12. Ni W, Yang X, Liu J, et al. Acute Myocardial Injury at Hospital Admission is Associated with All-cause Mortality in COVID-19 [published online ahead of print, 2020 May 8]. J Am Coll Cardiol. 2020; S07351097(20)35225-6. doi:10.1016/j.jacc.2020.05.007

13. Pei G, Zhang Z, Peng J, et al. Renal Involvement and Early Prognosis in Patients with COVID-19 Pneumonia. J Am Soc Nephrol. 2020;31(6):1157-1165. doi:10.1681/ASN.2020030276

14. Guan WJ, Ni ZY, Hu Y, et al. Clinical Characteristics of Coronavirus Disease 2019 in China. N Engl J Med. 2020;382(18):1708-1720. doi:10.1056/NEJMoa2002032

15. Yang $\mathrm{K}$, Sheng $\mathrm{Y}$, Huang $\mathrm{C}$, et al. Clinical characteristics, outcomes, and risk factors for mortality in patients with cancer and COVID-19 in Hubei, China: a multicentre, retrospective, cohort study [published online ahead of print, 2020 May 29]. Lancet Oncol. 2020;S1470-2045(20)30310-7. doi:10.1016/S14702045(20)30310-7

16. National Health Commission of the People's Republic of China. Chinese management guideline for COVID19 (version 5.0).

17. COVID-19 Treatment Guidelines Panel. Coronavirus Disease 2019 (COVID-19) Treatment Guidelines. National Institutes of Health. Available at https://www.covid19treatmentguidelines.nih.gov/. Accessed on May 30, 2020.

18. Damman K, Perez AC, Anand IS, et al. Worsening renal function and outcome in heart failure patients with preserved ejection fraction and the impact of angiotensin receptor blocker treatment. J Am Coll Cardiol. 2014;64(11):1106-1113. doi:10.1016/j.jacc.2014.01.087

19. Wang D, Yin Y, Hu C, et al. Clinical course and outcome of 107 patients infected with the novel coronavirus, SARS-CoV-2, discharged from two hospitals in Wuhan, China. Crit Care. 2020;24(1):188. Published 2020 Apr 30. doi:10.1186/s13054-020-02895-6

20. Preacher KJ, Hayes AF. Asymptotic and resampling strategies for assessing and comparing indirect effects in multiple mediator models. Behav Res Methods. 2008;40(3):879-891. doi:10.3758/brm.40.3.879

21. Zhou F, Yu T, Du R, et al. Clinical course and risk factors for mortality of adult inpatients with COVID-19 in Wuhan, China: a retrospective cohort study [published correction appears in Lancet. 2020 Mar 28;395(10229):1038] [published correction appears in Lancet. 2020 Mar 28;395(10229):1038]. Lancet. 2020;395(10229):1054-1062. doi:10.1016/S0140-6736(20)30566-3

22. Garg S, Kim L, Whitaker M, et al. Hospitalization Rates and Characteristics of Patients Hospitalized with Laboratory-Confirmed Coronavirus Disease 2019 - COVID-NET, 14 States, March 1-30, 2020. MMWR Morb Mortal Wkly Rep. 2020;69(15):458-464. Published 2020 Apr 17. doi:10.15585/mmwr.mm6915e3

23. Comorbidities, and Outcomes Among 5700 Patients Hospitalized With COVID-19 in the New York City Area [published online ahead of print, 2020 Apr 22] [published correction appears in doi:

10.1001/jama.2020.7681]. JAMA. 2020;323(20):2052-2059. doi:10.1001/jama.2020.6775 
24. Wang X, Fang X, Cai Z, et al. Comorbid Chronic Diseases and Acute Organ Injuries Are Strongly Correlated with Disease Severity and Mortality among COVID-19 Patients: A Systemic Review and MetaAnalysis. Research (Wash D C). 2020; 2020:2402961. Published 2020 Apr 19. doi:10.34133/2020/2402961

25. Naicker S, Yang CW, Hwang SJ, Liu BC, Chen JH, Jha V. The Novel Coronavirus 2019 epidemic and kidneys. Kidney Int. 2020;97(5):824-828. doi:10.1016/j.kint.2020.03.001

26. Rabb H. Kidney diseases in the time of COVID-19: major challenges to patient care. J Clin Invest. 2020;130(6):2749-2751. doi:10.1172/JCI138871

27. Matricardi PM, Dal Negro RW, Nisini R. The first, holistic immunological model of COVID-19: implications for prevention, diagnosis, and public health measures [published online ahead of print, 2020 May 2]. Pediatr Allergy Immunol. 2020;10.1111/pai.13271. doi:10.1111/pai.13271 Groß S, Jahn C, Cushman S, Bär C, Thum T. SARS-CoV-2 receptor ACE2-dependent implications on the cardiovascular system: From basic science to clinical implications [published online ahead of print, $2020 \mathrm{Apr} 30$ ]. J Mol Cell Cardiol. 2020; 144:47-53. doi:10.1016/j.yjmcc.2020.04.031

28. Groß S, Jahn C, Cushman S, Bär C, Thum T. SARS-CoV-2 receptor ACE2-dependent implications on the cardiovascular system: From basic science to clinical implications [published online ahead of print, 2020 Apr 30]. J Mol Cell Cardiol. 2020; 144:47-53. doi:10.1016/j.yjmcc.2020.04.031

29. Saavedra JM. Angiotensin receptor blockers and COVID-19. Pharmacol Res. 2020; 156:104832. doi:10.1016/j.phrs. 2020.104832

30. Kuba K, Imai Y, Rao S, et al. A crucial role of angiotensin converting enzyme 2 (ACE2) in SARS coronavirus-induced lung injury. Nat Med. 2005;11(8):875-879. doi:10.1038/nm1267

31. Frost DW, Shah R, Melvin L, et al. Principles for clinical care of patients with COVID-19 on medical units [published online ahead of print, 2020 Jun 3]. CMAJ. 2020;cmaj.200855. doi:10.1503/cmaj.200855

\section{Tables}

Table 1 Baseline characteristics of the included COVID-19 patients. 


\begin{tabular}{|ll|}
\hline Variables & All patients(n=1266) \\
\hline Age, years & $60.31(14.95)$ \\
\hline Gender, male & $606 / 1266(47.87 \%)$ \\
\hline Systolic blood pressure, mmHg & $130.52(17.39)$ \\
\hline Diastolic blood pressure, mmHg & $79.33(10.66)$ \\
\hline Smoker & $67 / 1266(5.29 \%)$ \\
\hline Comorbidities & \\
\hline Hypertension & $432 / 1266(34.12 \%)$ \\
\hline Cardiovascular disease & $208 / 1266(16.43 \%)$ \\
\hline Chronic kidney disease & $83 / 1266(6.56 \%)$ \\
\hline Diabetes & $187 / 1266(14.77 \%)$ \\
\hline Liver disease & $107 / 1266(8.45 \%)$ \\
\hline COPD & $192 / 1266(15.17 \%)$ \\
\hline Lab test & \\
\hline Infection-related markers & \\
\hline White blood cell count, *10 9/L & $5.56(2.42)$ \\
\hline Lymphocyte count, *10 9/L & $1.24(0.58)$ \\
\hline Leukocyte count, *10 9/L & $3.81(2.32)$ \\
\hline Platelet count, *10 9/L & $215.28(87.03)$ \\
\hline C reactive protein, mg/L & $9.93(1.48-44.44)$ \\
\hline Liver function & \\
\hline Alanine aminotransferase, IU/L & $24(15-38)$ \\
\hline Aspartate aminotransferase, IU/L & $26(20-37)$ \\
\hline Lactic dehydrogenase, IU/L & $201(161-272)$ \\
\hline Myocardial markers & \\
\hline Creatine kinase, IU/L & $68(45-119)$ \\
\hline Creatine kinase-MB, IU/L & \\
\hline Myoglobin, ng/ml & \\
\hline hs-TNT I, ng/ml & \\
\hline NT-proBNP, & \\
\hline Renal function & \\
\hline
\end{tabular}

Page 13/18 


\begin{tabular}{|ll|}
\hline Urea nitrogen, $\mathrm{mmol} / \mathrm{I}$ & $4.25(3.40-5.60)$ \\
\hline Baseline creatinine, umol/I & $63.60(51.60-79.50)$ \\
\hline Peak creatinine, umol// & $66.35(54.40-83.40)$ \\
\hline Decline of renal function & \\
\hline Decline $<10 \%$ & $1058 / 1266(83.57 \%)$ \\
\hline Decline $10-50 \%$ & $156 / 1266(12.32 \%)$ \\
\hline Decline $>50 \%$ & $52 / 1266(4.11 \%)$ \\
\hline
\end{tabular}

Data are presented as mean (SD), median (IQR) or number/total (percentage).

Abbreviation: COPD, chronic obstructive pulmonary disease; hs-TNT I, hypersensitivity troponin I; NT-proBNP, Nterminal pro-B-type natriuretic peptide.

Table 2 Logistic regression analysis between different comorbidities and the adverse outcomes.

\begin{tabular}{|lllllll|}
\hline Comorbidities & Critical cases & \multicolumn{3}{c|}{ ICU admission } & \multicolumn{3}{l|}{ In-hospital death } \\
\cline { 2 - 7 } & OR $(95 \% \mathrm{Cl})$ & $\begin{array}{l}\mathrm{P} \\
\text { value }\end{array}$ & OR $(95 \% \mathrm{Cl})$ & $\begin{array}{l}\mathrm{P} \\
\text { value }\end{array}$ & OR $(95 \% \mathrm{Cl})$ & $\begin{array}{l}\mathrm{P} \\
\text { value }\end{array}$ \\
\hline Hypertension & $1.31(0.90,1.90)$ & 0.15 & $1.14(0.71,1.83)$ & 0.59 & $1.43(0.81,2.54)$ & 0.22 \\
\hline CVD & $3.33(2.21,5.00)$ & $<.001$ & $2.93(1.77,4.86)$ & $<0.001$ & $6.12(3.35,11.19)$ & $<0.001$ \\
\hline CKD & $2.95(1.70,5.13)$ & $<.001$ & $3.04(1.61,5.75)$ & 0.001 & $7.08(3.55,14.13)$ & $<0.001$ \\
\hline Diabetes & $1.32(0.85,2.07)$ & 0.22 & $1.26(0.72,2.22)$ & 0.42 & $1.54(0.80,2.96)$ & 0.19 \\
\hline Liver disease & $1.47(0.83,2.63)$ & 0.19 & $2.51(1.36,4.64)$ & 0.003 & $2.44(1.12,5.31)$ & 0.02 \\
\hline COPD & $6.92(4.57,10.46)$ & $<0.001$ & $7.72(4.79,12.43)$ & $<0.001$ & $44.70(20.71,96.48)$ & $<0.001$ \\
\hline
\end{tabular}

Abbreviation: ICU, intensive care unit; $\mathrm{OR}$, odds ratio; $\mathrm{Cl}$, confidence interval; CVD, cardiovascular disease; CKD, chronic kidney disease; COPD, chronic obstructive pulmonary disease. All models were adjusted for age, gender, smoking status and white blood cell count.

\section{Figures}




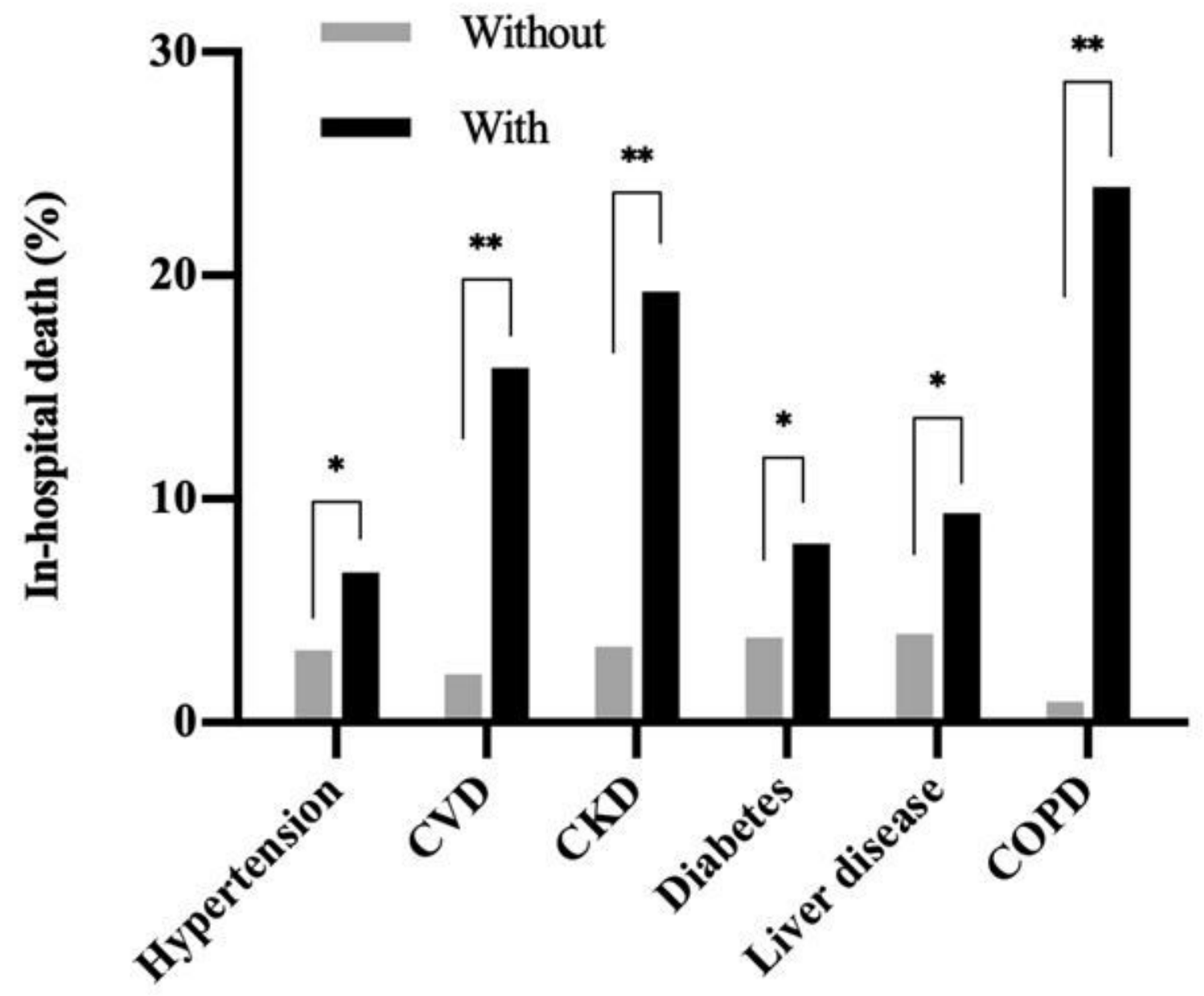

Figure 1

In-hospital death rate in patients with or without specific comorbidities. Patients with comorbidities had higher in-hospital death rates following COVID-19 infection (6.71\% for hypertension, 15.87\% for CVD, $19.28 \%$ for CKD, $8.02 \%$ for diabetes, $9.35 \%$ for liver disease and $23.96 \%$ for COPD). * indicates a P value $<0.05$ while ** indicates a $P$ value $<0.001$ between groups. 
A

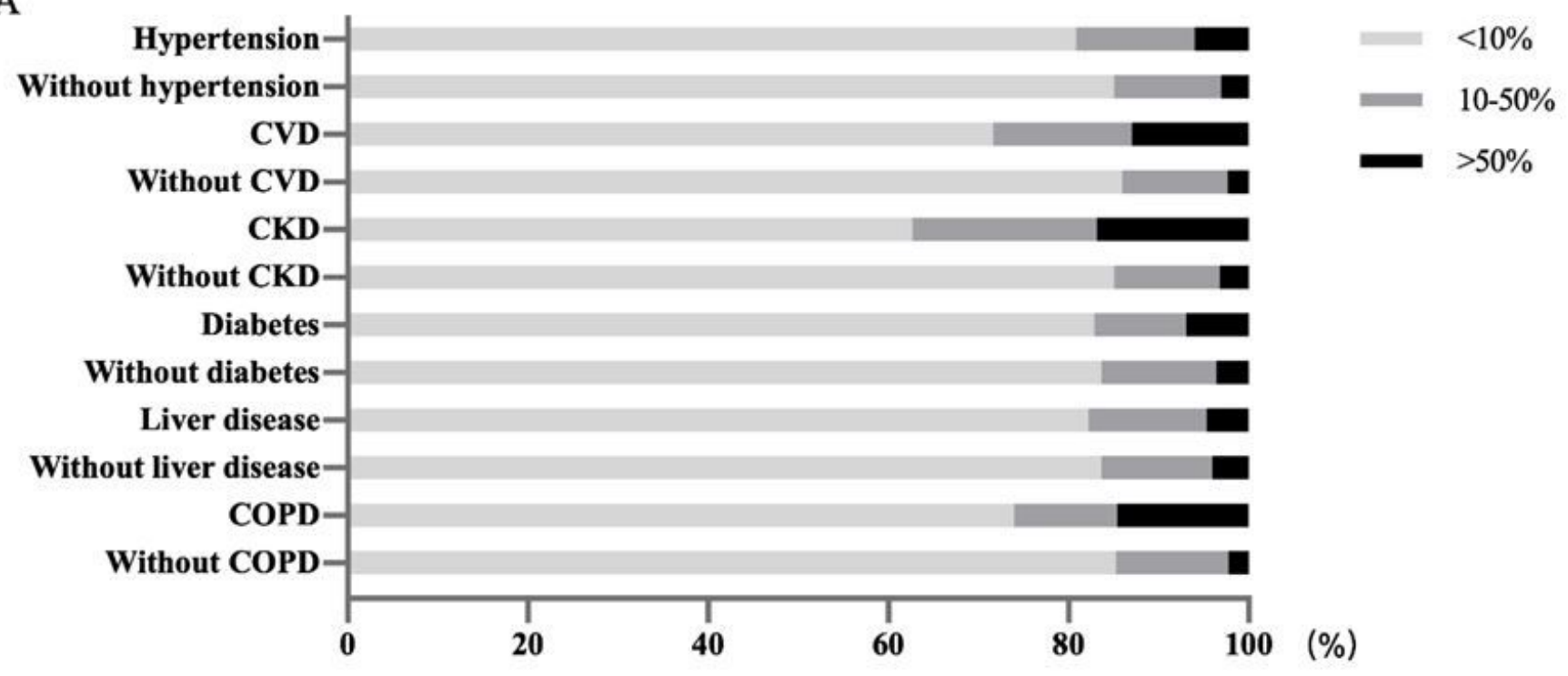

B

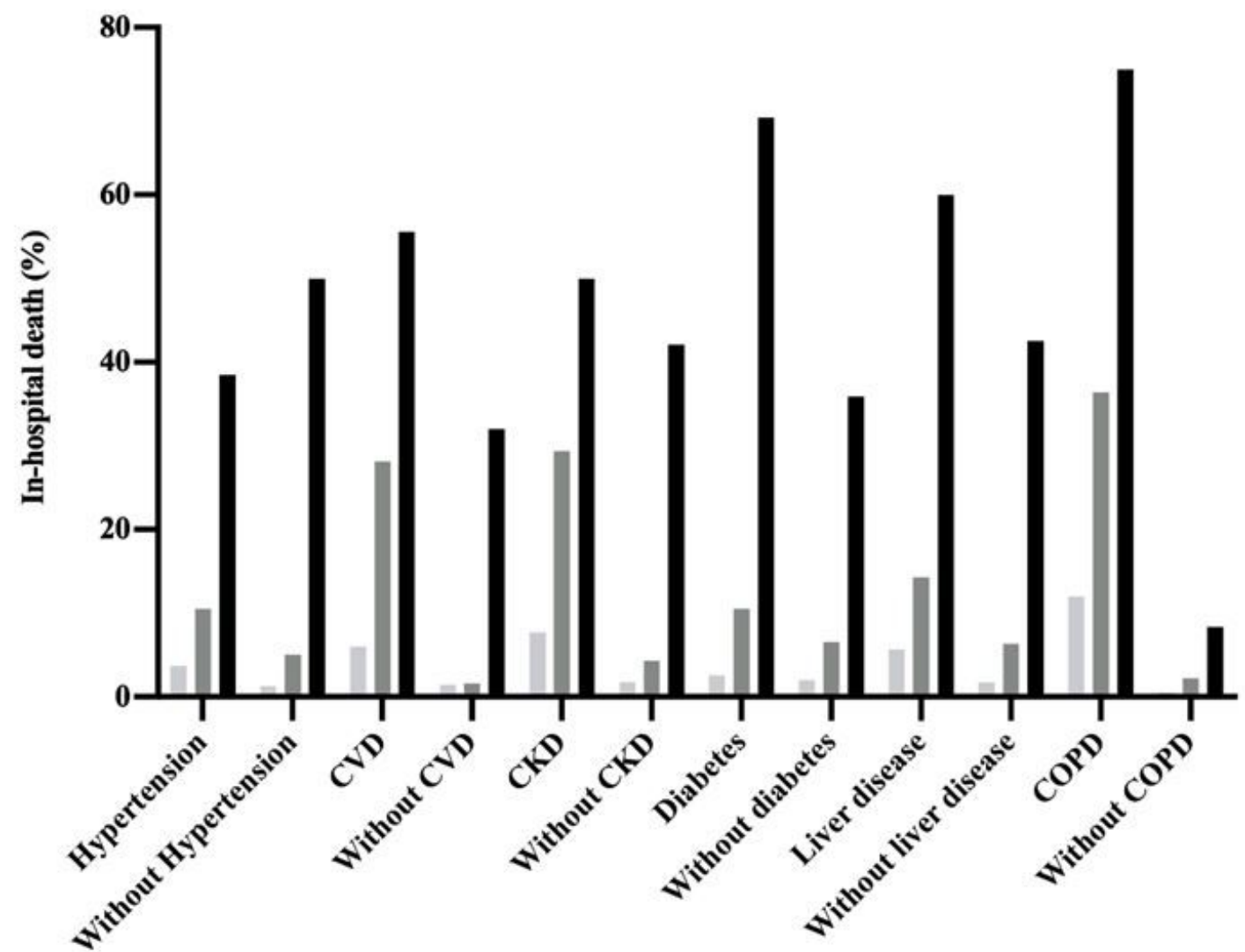

Figure 2

Distribution of deteriorated kidney function and in-hospital death in patients with or without comorbidities according to the decline of kidney function. A. Compared to patients without comorbidities, those with hypertension, CVD, CKD, diabetes, liver disease or COPD had correspondingly higher prevalence of more severe decline of kidney function. B. With similar degree of kidney function decline, the death rate was significantly higher in those with comorbidities. 


\begin{tabular}{|c|c|c|c|}
\hline $\begin{array}{l}\text { Deterioration of } \\
\text { kidney function }\end{array}$ & $\mathrm{OR}(95 \% \mathrm{CI})$ & $P$ value & \\
\hline \multicolumn{4}{|c|}{ Decline of kidney function, per unit } \\
\hline Model 1 & $4.27(2.85,6.41)$ & $<0.001$ & $\longmapsto$ \\
\hline Model 2 & $3.70(2.49,5.50)$ & $<0.001$ & $\longmapsto$ \\
\hline Model 3 & $3.85(2.52,5.88)$ & $<0.001$ & $\longmapsto$ \\
\hline \multicolumn{4}{|c|}{ Decline of kidney function } \\
\hline Decline $<10 \%$ & Reference & & \\
\hline \multicolumn{4}{|c|}{ Decline $10-50 \%$} \\
\hline Model 1 & $3.57(1.70,7.52)$ & 0.001 & $\longmapsto$ \\
\hline Model 2 & $3.51(1.65,7.49)$ & 0.001 & $\longmapsto$ \\
\hline Model 3 & $3.67(1.68,8.02)$ & 0.001 & $\longmapsto$ \\
\hline \multicolumn{4}{|l|}{ Decline $>50 \%$} \\
\hline Model 1 & $37.45(18.71,74.55)$ & $<0.001$ & $\longmapsto$ \\
\hline Model 2 & $29.29(14.38,59.68)$ & $<0.001$ & $\longmapsto$ \\
\hline Model 3 & $24.88(11.74,52.74)$ & $<0.001$ & $\longmapsto$ \\
\hline
\end{tabular}

Figure 3

Logistic regression analysis between deteriorated kidney function and in-hospital death in COVID-19 patients. Model 1 represented a non-adjusted model. Model 2 was adjusted for age and gender. Model 3 was adjusted for age, gender, smoking status, white blood cell count and baseline serum creatinine. OR, odds ratio. $\mathrm{Cl}$, confidence interval. 


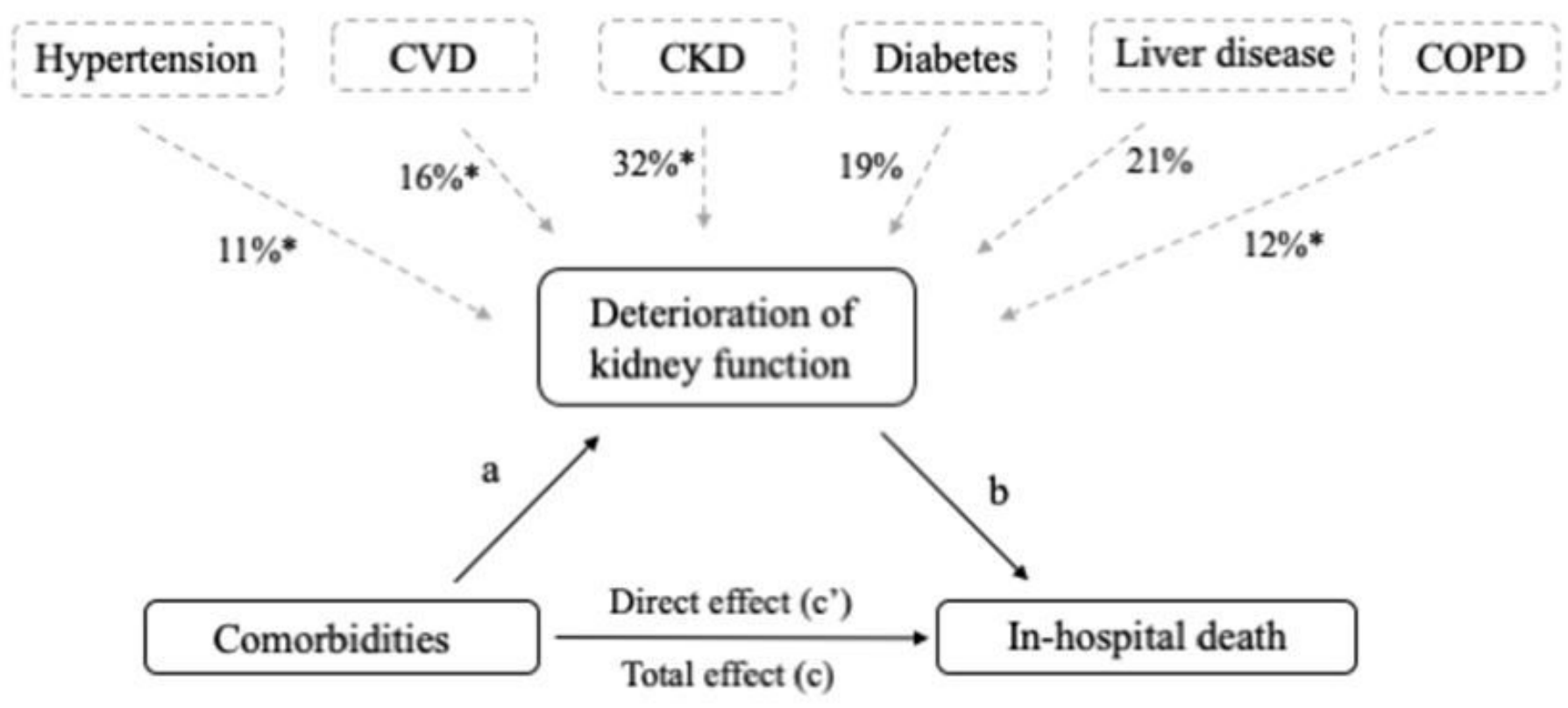

\section{Figure 4}

The mediation models between individual comorbidity and in-hospital death in COVID-19 patients. Pathway a represents the association between individual comorbidity and deteriorated kidney function. Pathway $b$ represents the association between deteriorated kidney function and in-hospital death in COVID-19 patients. The total proportion of overall effect mediated by deterioration of kidney function was $11 \%, 16 \%, 32 \%, 19 \%$, $21 \%$ and $12 \%$, respectively for hypertension, CVD, CKD, diabetes, liver disease and COPD. * indicates a significant mediation effect. 